\title{
Differences between left- and right-handers in approach/avoidance motivation: influence of consistency of handedness measures
}

\section{Scott M. Hardie* and Lynn Wright}

Evolutionary and Biological Approaches to Behaviour Research Group, School of Social and Health Sciences, University of Abertay, Dundee, UK

\section{Edited by:}

Onur Gunturkun, Ruhr-University

Bochum, Germany

Reviewed by:

Sven-Erik Fernaeus, Karolinska

Institutet, Sweden

Dirk Koester, Bielefeld University, Germany

\section{*Correspondence:}

Scott M. Hardie, Evolutionary and Biological Approaches to Behaviour Research Group, School of Social and Health Sciences, University of Abertay, Bell Street, Dundee, DD1 $1 \mathrm{HG}$, UK

e-mail:s.hardie@abertay.ac.uk
Hand preference is often viewed as a troublesome variable in psychological research, with left-handers routinely excluded from studies. Contrary to this, a body of evidence has shown hand preference to be a useful variable when examining human behavior. A recent review argues that the most effective way of using handedness as a variable, is a comparison between individuals who use their dominant hand for virtually all manual activities (consistent handers) versus those who use their other hand for at least one activity (inconsistent handers). The authors contend that researchers should only focus on degree of handedness rather than direction of preference (left versus right). However, we argue that the field suffers from a number of methodological and empirical issues. These include a lack of consensus in choice of cut-off point to divide consistent and inconsistent categories and importantly a paucity of data from left-handers. Consequentially, researchers predominantly compare inconsistent versus consistent right-handers, largely linked to memory, cognition and language. Other research on response style and personality measures shows robust direction of handedness effects. The present study examines both strength and direction of handedness on self-reported behavioral inhibition system (BIS) and behavioral activation system (BAS) scores, using evidence from a large $(N=689)$ dataset including more than 200 left-handers. There were degree of handedness effects on BIS and BAS-Fun Seeking, but effects are largely driven by differences between consistent left-handers and other groups. Choice of cut-off point substantively influenced results, and suggests that unless a suitable sample of left-handers is included, researchers clarify that their degree of handedness effects are applicable only to right-handers. We concur that strength of hand preference is an important variable but caution that differences related to consistency may not be identical in right and left-handers.

\section{Keywords: EHI, consistency, BIS/BAS, left-handed, inhibition, handedness strength, handedness direction}

\section{INTRODUCTION}

Hand preference has long been viewed as a troublesome variable in much research in psychology; left-handers in particular have been perceived as a noisy and unpredictable population and have often been excluded from studies (e.g., Ferrucci et al., 2013). However, a growing body of evidence suggests that handedness may provide some useful insights into individual differences in behavior (e.g., Wright et al., 2004; Kaploun and Abeare, 2010). Early research tended to examine differences between left- and right-handers and often found contradictory influences on behavior, although more recently there has been a move toward examining how handedness categories may influence the relationship with other variables (Beratis et al., 2011; Wright and Hardie, 2012; Hardie and Wright, 2013). In parallel, there is a growing body of research which focuses on strength of handedness, that is, the extent to which individuals favor their chosen hand (regardless of direction of preference). As a consequence, there has been debate in the literature about which of these aspects of handedness researchers should focus upon. The debate has been brought into focus by a recent review. This work contends that the most appropriate way to view handedness is using comparisons between consistent/strong handers who use their chosen hand for virtually all manual activities and inconsistent/mixed handers, who use their other hand for at least one activity (Prichard et al., 2013). Prichard et al. (2013) clearly advocate that "Instead of direction of hand preference being the variable of interest, it should be degree" (p. 1).

While Prichard et al. (2013) provide a very useful synthesis of strength of handedness research and expand our understanding of handedness (especially in the areas of memory retrieval and belief updating/cognitive flexibility); there are problems with the notion that direction is not an appropriate measure. For example, on the basis of an item response theory evaluation of one of the major handedness questionnaires (Oldfield's, 1971; Edinburgh Handedness Inventory, EHI), Büsch et al. (2010) strongly argue that only two classes of response emerge - left- and righthanded. Other researchers have argued that the most appropriate way to measure handedness is to examine a tripartite model - covering consistent-left, consistent-right and mixed-handers, based on factor-analysis (Peters and Murphy, 1992), neuroimaging (e.g., Knecht et al., 2000; Kirveskari et al., 2006) and behavioral studies 
(Kaploun and Abeare, 2010). Finally, recent work in this area combines both direction and degree; Lyle et al. (2012a) found differences between consistency groups within handedness categories. This is potentially the most effective way of understanding the influence of handedness on behavior, and may be seen as a "gold standard" for future work, but such studies remain rare.

In order for degree of handedness to be considered as a valid variable, there is a need to examine the empirical and conceptual basis for this measure. After conducting a review of the methodology and theoretical stance of the authors of more than 30 articles using consistency of handedness as a variable, four major issues were identified. The first two are issues which are important for the field to debate and come to a consensus over, and are noted here simply in order to stimulate debate, while the second two issues will be empirically examined.

Firstly, the use of the EHI as a measure of handedness may be criticized. Oldfield's, (1971) EHI is a self-report measure and respondents answer questions regarding their preference to use a chosen hand "always" "mostly" or to use "either" hand on 10 manual tasks (e.g., writing, throwing). Scores are converted into a Laterality Quotient ranging from -100 (complete left-hand preference) through 0 (no preference) to +100 (complete right-hand preference). This instrument has been extensively evaluated since its' inception (Bryden, 1977; Williams, 1986; Dragovic, 2004), highlighting problems with the original scoring system (Fazio et al., 2012) which can lead to errors, as well as issues with the structure of the questionnaire itself (Dragovic, 2004). Recently, Milenkovic and Dragovic (2013) proposed that a seven-item version was superior to the original 10 -item version, although Veale (2014) disputes this, instead offering her own four-item version. The crux of this debate is that some items may cause considerable measurement error, and that the 10 -item version may lead to an overestimation of the proportion of mixed-handers (Dragovic et al., 2008; Büsch et al., 2010). The field needs to address these problems and agree on a standardized way to measure hand preference strength, before an accurate assessment of findings can be made.

Secondly, the use of a split to divide a potentially continuous variable (in this case, strength of handedness) into discrete categories has been criticized for at least the last 30 years (Cohen, 1983; Streiner, 2002; Irwin and McClelland, 2003). MacCallum et al. (2002), for example, argue that it can result in a loss of analytical power or may create falsely significant results. DeCoster etal. (2009) specifically examined the use of dichotomization of samples in psychological research, contacting a number of researchers to establish their rationale for this. The researchers followed this up with Monte Carlo simulations and conclude that continuous variables outperformed dichotomized versions in the majority, but not all of the cases. They produced criteria for dichotomising samples, but it should be noted that the emphasis was on the use of data to support the categorization process. This poses a question for researchers, if dichotomization is being used, should it be done on a seemingly artificial basis (median of hand strength), or should it rely on a split based on underlying latent classes (such as left versus right Büsch et al., 2010)? Another option might be to use the mean score in each sample and convert the preference scores into stanines, and use stanine- 5 exclusion to split the sample. This type of split is used in some areas of psychology (e.g., Moritz et al., 2006) and may be worthy of examination. Alternatively, DeCoster et al. (2009) suggest that extreme group analysis (i.e., selectively recruiting participants from the extremes) is a viable strategy, so perhaps this might be a useful way of testing consistency? The use of a median split for dichotomization needs to be more strongly justified by researchers, perhaps using DeCoster et al. (2011) recommendations.

Assuming that handedness should be examined using a noncontinuous categorization, the third issue relates to the choice of the cut-off point to divide populations into consistent versus inconsistent handers (IH). The majority of studies use a notional median value of 80 on the EHI to split their groups into consistent and inconsistent (e.g., Christman and Butler, 2011; Lyle and Orsborn, 2011; Dollfus et al., 2012; Westfall et al., 2012), but it is not clear whether this median value is consistently found within individual samples. It is not common practice in many of these studies to publish their own median values, making the validity of a median split at 80 questionable. Even if the median value is established, there are additional problems with a lack of consistency in how to operationalize the split itself. For example, there are times when the consistency group is defined as scoring above the proposed median, i.e., 85 or above (Propper et al., 2005; Christman et al., 2009; Jasper et al., 2009). There are other times when it is defined as scoring at the median and above, i.e., 80 or above (Christman et al., 2006; Lyle and Grillo, 2014), or occasionally at some other figure such as 95 or above (e.g., Lyle et al., 2008). This lack of consistency across studies makes it difficult to directly compare findings and also suggests that choice of cut-off may influence results.

The final, and arguably the most important issue relates to how left-handers fit into this area of research. As a group, lefthanders present a challenge to researchers, as they are generally less strongly lateralized than right-handers (Oldfield, 1971) and are relatively scarce, comprising approximately $10 \%$ of the general population (Ellis etal., 1988). Even more problematic, is that consistent or strongly lateralized (EHI <-80) left-handers make up only 2-3\% of all individuals (Prichard etal., 2013). This makes them an extremely difficult group of participants to recruit, and only a few of the many studies of degree of handedness have been able to recruit a sufficient number of strong left-handers to be able to examine them as a group. As noted by Prichard etal. (2013) this means that the vast majority of this research predominantly compares inconsistent versus consistent groups largely, or exclusively, made up of right-handers (for an exception, see Lyle etal., 2012b). This conflicts with much of the literature which states their findings in terms of consistent versus IH, without making the rightward bias clear in terms of the narrative used in title, introduction and discussion (e.g., Niebauer, 2004; Westfall et al., 2010; Rose and Nagel, 2012). By failing to have enough data on strong left-handers, researchers are not in a sufficiently robust position to be able to say whether they are definitely the same as, or different from, strong right-handers. To clarify this, it is suggested that the field states clearly when the comparison group is predominantly made 
up of right-handers only (for an example of this approach, see Christman, 2013).

The current study seeks to examine the issues of cut-off point choice and a lack of empirical data from left-handers, in light of Prichard et al.'s (2013) review and their strong assertion that direction of handedness is a "more powerful and appropriate way to classify handedness than the traditional one based on direction (right versus left)" (p. 1). Arguably this assertion is premature, particularly due to a lack of data from consistent left (CL)-handers, and that the studies thus far suffer from a lack of agreement in terms of the cut-off points used to test consistency effects. Consequentially, there is one main research question that requires answering: does strength of handedness influence leftand right-handers in the same way?

In order to do this, the present research examines the influence that strength of handedness has on a dataset which has a relatively large $(N=202)$ number of left-handers, seeking to understand the potential relationship both strength and direction may have on findings. As noted previously, recent work on degree of handedness has been extensively linked to areas of cognition such as memory. The present study extends this into an area of personality, focusing on the relationship between handedness and motivation measured by the behavioral inhibition system (BIS) and behavioral activation system (BAS) scales of Carver and White (1994). The BIS/BAS scales are a self-report measure of the revised reinforcement sensitivity theory of personality (rRST; Gray and McNaughton, 2000). Briefly, this theory postulates that behavior is broadly influenced by three interacting systems; the BAS which motivates approach, reward and impulsivity; the fight-flight-freeze system (FFFS) which relates to fear of a negative outcome, punishment and withdrawal; and the BIS which resolves conflict within or between the other two systems (see Corr and McNaughton, 2008 for details). Prior studies have linked the right-hemisphere to behavioral inhibition and behavioral avoidance (e.g., Davidson, 1985, 1995, 1998), with Sutton and Davidson (1997) describing the left hemisphere as corresponding to approach behavior and the right hemisphere to avoidance behavior. For example, Shackman etal. (2009) have shown that individuals reporting themselves as behaviorally inhibited have an increased resting activity within their right dorsolateral prefrontal cortex. Other work links the right-hemisphere to infants' temperamental shyness, anxiety, and behavioral inhibition (Schmidt et al., 1999; Fox et al., 2001). Added to this are animal studies linking lefthand preference to delays in exploratory and investigative behavior (Hopkins and Bennett, 1994; Cameron and Rogers, 1999). There is evidence to suggest that measurements of lateral preferences are indicators of hemispheric preferences (Kinsbourne, 1997; Jackson, 2008), with the lateral preference indicative of a preference for the contralateral hemisphere. Previous work in this area (Wright et al., 2009) found hand direction differences, but the evidence has yet to be examined in terms of strength of handedness.

The current study seeks to investigate whether strength of handedness influences left- and right-handers in the same way, in terms of their relationship to BIS/BAS variables. This will be investigated empirically in two ways:
(1) Systematically examining the influence of cut-off points on strength of handedness findings. This will be done by comparing the dataset using a range of cut-off points derived from both the literature and the data itself.

(2) Examining how strength of handedness relates to other variables? This will use regression to examine whether the relationship between variables is the same for both handedness groups.

\section{MATERIALS AND METHODS PARTICIPANTS}

Six hundred and eighty-nine participants took part in this study, 272 were male and 417 were female. The majority $(N=502)$ were in the 18-29 year category, comprising $76 \%$ of males and $71 \%$ of females. Two hundred and two were left-handed, 481 were right-handed, and the remaining six had no overall preference.

\section{MEASURES}

Demographics including gender and age category (18-29, 30-39, $40-49,50-59,60-69$, and $70+$ years) were collected. The EHI (Oldfield, 1971) was used to measure strength and direction of handedness, where participants were asked to indicate which hand they would normally use in each of ten tasks. Choices were Left Always, Left Mostly, Either, Right Mostly, Right Always, and as in previous work (Hardie and Wright, 2013) these were scored as $-10,-5,0,5$, and 10 , respectively. Totaling this up yielded a score ranging from -100 (completely left-handed) to +100 (completely right-handed).

Carver and White's (1994) BIS/BAS scale was used to measure self-reported BIS, BAS and FFFS scores. This instrument has 20 items sub-divided into four categories. Three scales measure BAS - Reward Responsiveness (e.g., "It would excite me to win a contest"), Fun Seeking (e.g., "I often act on the spur of the moment") and Drive (e.g., "I go out of my way to get things I want". Originally only a single category measured BIS sensitivity (e.g., "Criticism, or scolding hurts me quite a bit") but this has subsequently been subdivided into FFFS (questions 2 and 22) and BIS scales (remaining five BIS questions) based on previous work (Corr and McNaughton, 2008; Hardie and Wright, 2013). In all cases, questions were answered as one of four options, ranging from "Very false for me" to "Very true for me" and scored as per Carver and White (1994).

\section{PROCEDURE}

Participants were recruited from both university and the general public through a sustained campaign of emails, website notices, recruitment at public science centers, and at science fairs over the course of around 12 months. We paid particular attention to the recruitment of left-handers, asking for people who considered themselves to be "left-handed" but we also recruited people more generally and tested all individuals who agreed to participate regardless of hand preference. Testing was carried out via a webbased presentation of the questionnaires, in a randomized order, after participants agreed to participate. The research was approved by the school research ethics committee and abided by the British Psychological Society Code of Human Research Ethics. 
Table 1 | Distribution of EHI scores.

\begin{tabular}{|c|c|c|c|c|c|c|c|c|}
\hline & \multicolumn{2}{|c|}{$\begin{array}{l}\text { Whole DATA set } \\
\qquad(N=689)\end{array}$} & \multicolumn{2}{|c|}{$\begin{array}{l}\text { Absolute strength } \\
\qquad(N=689)\end{array}$} & \multicolumn{2}{|c|}{$\begin{array}{l}\text { Left-handers only } \\
\qquad(N=202)^{*}\end{array}$} & \multicolumn{2}{|c|}{$\begin{array}{l}\text { Right-handers only } \\
\qquad(N=481)^{*}\end{array}$} \\
\hline \multirow[t]{2}{*}{ EHI score mean (SD) } & \multicolumn{2}{|c|}{$32.43(64.1)$} & \multicolumn{2}{|c|}{$67.0(25.8)$} & \multicolumn{2}{|c|}{$-59.0(25.5)$} & \multicolumn{2}{|c|}{$71.22(24.1)$} \\
\hline & $\begin{array}{c}\text { Male } \\
(N=272)\end{array}$ & $\begin{array}{c}\text { Female } \\
(N=417)\end{array}$ & $\begin{array}{c}\text { Male } \\
(N=272)\end{array}$ & $\begin{array}{c}\text { Female } \\
(N=417)\end{array}$ & $\begin{array}{l}\text { Male Left } \\
(N=86)\end{array}$ & $\begin{array}{l}\text { Female Left } \\
(N=116)\end{array}$ & $\begin{array}{l}\text { Male Right } \\
(N=186)\end{array}$ & $\begin{array}{l}\text { Female Right } \\
(N=295)\end{array}$ \\
\hline EHI score median & 60 & 60 & 70 & 70 & -65 & -65 & 75 & 75 \\
\hline EHI score mean (SD) & $29.9(65.5)$ & $34.1(63.2)$ & $68.1(23.0)$ & $66.3(27.4)$ & $-60.5(24.4)$ & $-57.8(26.3)$ & 71.6 (21.5) & $71.0(25.6)$ \\
\hline
\end{tabular}

*Six individuals who had an EHI score of 0 were removed for the left versus right figures.

\section{STATISTICAL ANALYSIS}

Statistical analyses were carried out using SPSS v21. $\alpha$ was set at 0.05 . The strength of hand preference scores were initially examined in terms of median scores, compared by direction of hand preference and gender. This was followed by an investigation of the influence of categorization system on measures of BIS/BAS, with all participants being assigned into Consistent/Inconsistent categories based on six separate classification systems with different cut-off points on the EHI. As gender has been shown to influence BIS/BAS scores, it was also included as a factor in all analyses. For each classification scheme the following three analyses were carried out:

Consistent handedness versus inconsistent handedness regardless of direction [ANOVA 2 (Gender) $\times 2$ (Consistency)].

Consistent left (CL, consistent right (CR), and inconsistent $(\mathrm{IH})$ handers [ANOVA 2 (Gender) $\times 3$ (Consistency)].

Consistent left (CL), inconsistent left (IL), consistent right (CR), and inconsistent right (IR) handers [ANOVA: 2 (Gender) $\times 4$ (Consistency) $]$.

This was undertaken on BIS, FFFS, BAS-Reward Responsiveness (BAS-RR), BAS-Fun Seeking (BAS-FS), BAS-Drive (BAS-D), as well as a combined BAS score. For the second and third analyses, post hoc tests with Bonferroni corrections were calculated where a main effect of consistency was found. Only significant results will be reported.
Handedness was also examined in terms of a regression model, undertaking the following regression analyses:

EHI scores to include directionality and absolute scores to assess general relationship to strength.

Left versus right (as has been used in other studies, such as Hardie and Wright, 2013) where the analyses look at each category separately.

As gender was related to most of these measures, stepwise multiple regressions were used to examine the relationship between handedness and BIS/BAS variables. For each of the analyses, step one was to regress the BIS/BAS measures on gender. In step two, the measures of hand strength were introduced. A significant increase in $R^{2}$ when comparing the first to second step would indicate that handedness accounts for variance in BIS/BAS measures over and above those related to gender. If EHI is a significant predictor, then direction of handedness is important, while if absolute is significant then strength is most important. Beta weights provide the basis for examining any relationships. The key data is $R^{2}$ change and individual beta weights for the variables of interest.

\section{RESULTS}

Table 1 shows that the cut-off for the entire sample is 60, for left-handers only, it is -65 and for right-handers only, it is 75 .

Table 2 | Influence of split value used, in terms of number of participants in each category.

Median categorization system Consistent left-handers Inconsistent left-handers Consistent right-handers Inconsistent right-handers

\begin{tabular}{lccc}
\hline EHI85 (Consistent \pm 85$)$ & $38(5.6 \%)$ & 164 & $184(26.9 \%)$ \\
EHI80 (Consistent \pm 80$)$ & $53(7.8 \%)$ & 149 & $235(34.4 \%)$ \\
EHI75 (Consistent \pm 75$)$ & $66(9.7 \%)$ & 136 & 246 \\
EHI70* (Consistent \pm 70$)$ & $87(12.7 \%)$ & 115 & $304(44.5 \%)$ \\
EHI60** (Consistent \pm 60$)$ & $119(17.4 \%)$ & 83 & $367(53.7 \%)$ \\
EHI40 (Strong \pm 45$)$ & $145(21.2 \%)$ & 57 & 174 \\
\end{tabular}

*Based on actual median calculated from absolute strength figures.

**Based on actual median calculated from EHI strength figures. 


\section{EXAMINATION OF THE INFLUENCE OF CATEGORIZATION SYSTEMS}

Table 2 shows variation within handedness categories depending upon the classification system used. The percentage of the sample categorized as consistent left - ranged from $5.6 \%$ of the sample in the most stringent to $21.2 \%$ in the loosest classification, and for CR-handers, these ranged from $26.9 \%$ (stringent) to $57.8 \%$ (loosest).

For all variables (except total BAS) gender was a significant factor. Females were significantly higher on BIS, FFFS and BAS-RR, while males were significantly higher on BAS-D and BAS-FS. All are $F_{(1,685)}>4.7$, with $p$ values $=0.035$ or lower. There were no interactions between gender and handedness categories. The remaining analyses, therefore, focus on the influence of categorization. There were no main effects using either the EHI60 or EHI40 classifications.

\section{TWO CATEGORY SPLITS: CONSISTENT (CH) VERSUS INCONSISTENT (IH) HANDERS}

Table 3 shows that $\mathrm{CH}$ had a significantly higher FFFS score only when using the EHI85 cut-off point $\left[F_{(1,685)}=12.17, p=0.001\right]$.

\begin{tabular}{|c|c|c|c|c|c|}
\hline Scale & Finding* & $\begin{array}{l}\text { EHI85 } \\
\text { Split }\end{array}$ & $\begin{array}{l}\text { EHI80 } \\
\text { Split }\end{array}$ & $\begin{array}{l}\text { EHI75 } \\
\text { Split }\end{array}$ & $\begin{array}{l}\text { EHI70 } \\
\text { Split }\end{array}$ \\
\hline \multicolumn{6}{|c|}{ Two categories } \\
\hline FFFS & $\mathrm{CH}>\mathrm{IH}$ & 0.001 & & & \\
\hline BIS & $\mathrm{CH}>\mathrm{IH}$ & 0.006 & 0.035 & 0.011 & \\
\hline BAS-FS & $\mathrm{IH}>\mathrm{CH}$ & 0.009 & 0.029 & 0.020 & \\
\hline \multicolumn{6}{|c|}{ Three categories } \\
\hline FFFS & $\mathrm{CR}>\mathrm{IH}$ & 0.002 & & & \\
\hline \multirow[t]{2}{*}{ BIS } & $\mathrm{CL}>\mathrm{IH}$ & 0.001 & 0.046 & 0.005 & 0.014 \\
\hline & $\mathrm{CL}>\mathrm{CR}$ & 0.018 & & & \\
\hline BAS-FS & $\mathrm{IH}>\mathrm{CL}$ & 0.005 & 0.011 & 0.007 & \\
\hline \multirow[t]{2}{*}{ BAS-RR } & $\mathrm{CL}>\mathrm{CR}$ & & & 0.037 & 0.009 \\
\hline & $\mathrm{CL}>\mathrm{IH}$ & & & & 0.046 \\
\hline \multicolumn{6}{|c|}{ Four categories** } \\
\hline \multirow[t]{2}{*}{ FFFS } & $\mathrm{CR}>\mathrm{IL}$ & 0.008 & & & \\
\hline & $\mathrm{CR}>\mathrm{IR}$ & 0.035 & & & \\
\hline \multirow[t]{3}{*}{ BIS } & $\mathrm{CL}>\mathrm{CR}$ & 0.036 & & & \\
\hline & $\mathrm{CL}>\mathrm{IL}$ & 0.010 & & & \\
\hline & $\mathrm{CL}>\mathrm{IR}$ & 0.001 & ns & 0.007 & 0.038 \\
\hline \multirow[t]{2}{*}{ BAS-FS } & $\mathrm{IL}>\mathrm{CL}$ & 0.019 & 0.028 & 0.017 & \\
\hline & $\mathrm{IR}>\mathrm{CL}$ & 0.013 & 0.037 & 0.031 & \\
\hline BAS-RR & $\mathrm{CL}>\mathrm{CR}$ & & & & 0.019 \\
\hline
\end{tabular}

${ }^{*} \mathrm{CH}$, consistent handers; $\mathrm{IH}$, inconsistent handers; $\mathrm{CL}$, consistent left-handers; $C R$, consistent right-handers; IL, inconsistent left-handers; IR, inconsistent righthanders.

** Six individuals who had an EHI score of 0 could not be assigned a direction (left or right) and were removed from the four category analyses.
Looking at BIS scores, $\mathrm{CH}$ had significantly higher values in the EHI85 $\left[F_{(1,685)}=7.73, p=0.006\right]$, EHI80 $\left[F_{(1,685)}=4.47\right.$, $p=0.035]$, and EHI75 $\left[F_{(1,685)}=6.49, p=0.011\right]$ classifications. IH had a significantly higher value of BAS-FS, and like the BIS scores, these were only significant in EHI85 $\left[F_{(1,685)}=6.87\right.$, $p=0.009]$, EHI80 $\left[F_{(1,685)}=4.78, p=0.029\right]$, and EHI75 $\left[F_{(1,685)}=5.46, p=0.020\right]$ classifications. There were no other significant effects.

\section{THREE CATEGORY SPLITS: CONSISTENT RIGHT (CR), CONSISTENT LEFT (CL) AND INCONSISTENT (IH) HANDERS}

There was a significant main effect of category on FFFS scores (EHI85), $F_{(2,683)}=6.23, p=0.002$. Post hoc analyses revealed that only CR was significantly higher than $\mathrm{IH}(p=0.002)$. For $\mathrm{BIS}$, there was an influence of category on differences from EHI85 through to EHI70, all $F_{(2,683)}>3.34$, $p$ values $=0.036$ or lower. Further analyses showed that CL was significantly different from CR in only the EHI85 system, but differed from IH in all classifications. For BAS-FS, there were main category effects in EHI85, EHI80 and EHI75 $-F_{(2,683)}>4.55$, with $p$ values $=0.011$ or lower. In all cases, only CL was significantly lower than IH. There was an additional main effect of categorization on BAS$\mathrm{RR}$, for both EHI75 $\left[F_{(2,683)}=3.24, p=0.040\right]$ and EHI70 $\left[F_{(2,683)}=4.44, p=0.012\right]$. In both cases CL were significantly higher than IH, and in EHI70 CL were also significantly higher than CR.

\section{FOUR CATEGORY SPLITS: CONSISTENT RIGHT (CR), CONSISTENT LEFT (CL), INCONSISTENT LEFT (IL) AND INCONSISTENT RIGHT (IR) HANDERS}

Comparing fully across hand direction and consistency categories helps to clarify where differences are arising (Table 3). Again the FFFS main effect is linked to the EHI85 classification only, $F_{(3,675)}=4.27, p=0.005$ and post hoc analyses revealed CR were significantly higher scoring than both IL and IR. The consistent groups did not significantly differ. For BIS, there were significant main effects of classification system from EHI85 through to EHI70, all with $F_{(3,675)}>2.66$, $p$ values $=0.047$ or lower. In all cases CL were significantly greater than IR, except for EHI80 $(p=0.052)$. For EHI85, CL were also significantly higher in BIS than CR and IL. There were also main effects of classification system on BAS-FS scores for EHI85, EHI80 and EHI75 categories [all $F_{(3,675)}>3.13$, $p$ values $=0.025$ or lower $]$. In all three cases, CL were significantly lower than both IL and IR. Finally, for EHI70 there was also a difference in BAS-RR scores, $F_{(3,675)}=3.07, p=0.027$, where only the CL group had significantly higher scores than CR $(p=0.019)$.

\section{RELATIONSHIP OF STRENGTH OF HAND PREFERENCE TO OTHER VARIABLES}

As the previous analyses predominantly found differences in BIS and BAS-FS then the following analyses are limited to these.

\section{STRENGTH OF HANDEDNESS}

In step 1 (Gender) the model successfully predicted BIS $\left[F_{(1,688)}=50.22, \quad p<0.0001, \quad R^{2}=0.07\right]$ and BAS-FS $\left[F_{(1,688)}=8.24, p=0.004, R^{2}=0.01\right]$ and was also the case in step 2 (Gender and Handedness), for both BIS $\left[F_{(3,688)}=19.85\right.$, 
$\left.p<0.0001, R^{2}=0.08\right]$ and BAS-FS $\left[F_{(3,688)}=4.11, p=0.007\right.$, $\left.R^{2}=0.02\right]$. In the case of BIS, the introduction of handedness in Step 2 significantly improved the model, $F_{(2,685)}=4.41, p=0.012$, $\Delta R^{2}=0.012$. Both EHI $[\beta=-0.088, t(688)=-2.26, p=0.024]$ and absolute strength $[\beta=0.101, t(688)=2.59, p=0.010]$ were significant but weak predictors of BIS. In the case of BAS-FS, handedness failed to significantly improve the model, and absolute strength was not a significant predictor. In general, handedness only explained a very small amount of overall variance.

\section{STRENGTH OF HANDEDNESS FOR EACH CATEGORY}

The sample was divided into left- and right-handers in order to investigate the relationship between BIS-measures and handedness strength separately. In this case, only absolute strength is used, as for each handedness group this is effectively the same figure.

\section{Right-handers (i.e., EHI scores $>0, N=481$ )}

Neither BIS nor BAS-FS were significantly correlated with absolute strength. The model successfully predicted BIS in both Step $1\left[F_{(1,480)}=47.96, p<0.0001, R^{2}=0.09\right]$ and Step 2 $\left[F_{(2,480)}=24.15, p<0.0001, R^{2}=0.09\right]$ but the introduction of strength of handedness in Step 2 did not significantly improve the model. The model failed to successfully predict BAS-FS.

\section{Left-handers (i.e., EHI scores $<0, N=202$ )}

BIS significantly correlated with absolute hand strength $r(202)=0.140, p=0.024$, but BAS-FS did not. BIS was successfully predicted in both Step $1\left[F_{(1,201)}=7.95, p=0.005\right.$, $\left.R^{2}=0.04\right]$ and Step $2\left[F_{(2,201)}=6.44, p=0.002, R^{2}=0.06\right]$ and the introduction of strength of handedness in Step 2 significantly improved the model $F_{(1,199)}=4.78, p=0.030, \Delta R^{2}=0.023$, with strength of handedness a significant predictor of BIS $[\beta=0.150$, $t(201)=2.19, p=0.030]$. BAS-FS was also successfully predicted in both Step $1\left[F_{(1,201)}=5.32, p=0.022, R^{2}=0.03\right]$ and Step $2\left[F_{(2,201)}=3.80, p=0.024, R^{2}=0.04\right]$ but introduction of handedness in Step 2 did not significantly improve the model.

\section{DISCUSSION}

The current study did not find a median hand preference score of 80, a value which has been used in most previous studies (e.g., Christman et al., 2008; Westfall et al., 2010; Lyle et al., 2012a). The present sample comprises a relatively large sample of lefthanders added to a sample of more than 400 right-handers, which yielded a median strength of 60 . By taking absolute score (strength regardless of direction) this increases, but even examining only right-handers there is a median of 75 . This calls into question the robustness of using a fixed value cut-off point based on a notional median score, and also illustrates the potential confound that may arise when using an actual versus notional median value. Differences in scoring of the EHI may potentially be a factor, as the original scoring system is problematic (Fazio et al., 2012). However, most researchers in the field appear to use a system dividing strength into multiples of 5 ranging from -10 for left-always to +10 for right-always (e.g., Christman et al., 2008; Lyle et al., 2012a; Hardie and Wright, 2013), so it is unlikely that scoring differences greatly influenced the current results.
Examining the influence of strength and direction of handedness on measures of Carver and White's (1994) BIS/BAS scales demonstrated that consistency of handedness had an influence on several measures. These were mainly related to BIS and BAS-FS which were significantly different across three different split points (EHI85, EHI80, and EHI75), suggesting that these were robust differences. As mentioned above, the current study did not find a median score of 80 , so the use of this as a cut-off point may be questioned. When using a notional median of 80 , which a majority of studies do (e.g., Jasper et al., 2008; Christman and Butler, 2011; Lyle and Orsborn, 2011; Westfall et al., 2012), the present study demonstrated some strong differences between $\mathrm{CH}$ and $\mathrm{IH}$ especially when using an "above median" cut-off of 85 (e.g., Propper et al., 2005; Jasper et al., 2009). For BIS scores, using the three category model (CR, CL, and IR) then CL were higher in BIS than the other two groups, suggesting that this group were strongly influencing the findings. In the four category classification (additionally splitting IH into IL and IR), CL were again significantly higher in BIS than the other three categories. Therefore, by selectively choosing this cut-off point to determine consistent handers, the current research findings could be interpreted as strongly arguing that consistent left-handers were significantly higher in BIS than other handedness groups and suggests that the increased behavioral inhibition of left-handers (e.g., Wright et al., 2009) may be driven by this group. This illustrates that when examined in a sufficient number, consistent left- and right-handers may differ from each other, supporting the contention that in the absence of enough data on left-handers other studies should not automatically assume similarity in behavior.

As expected, it was clear that the choice of cut-off point influenced the extent to which consistency effects on BIS/BAS were shown. This is an important finding, as comparing across other handedness consistency research there is a range of cut-off values for defining the "consistent hander" group, mainly equivalent to EHI85, EHI80, and EHI75 classifications used here. The present work is the first demonstration of the direct effect of choice of how to operationalize the median split point within the same dataset, highlighting the influence and also the need for consensus. A wider examination of the use of median splits across psychology yields a similarly mixed picture. A large proportion of them are largely silent in terms of how a median split is operationalized, for example, indicating that the variable in question was split into two groups based on the median, but not stating what was done with those at the median (e.g., Rydstedt et al., 2008; Tops and Bokshem, 2011; McCullough et al., 2012; Smith et al., 2013). It is common practice to have the split at a point scoring above the median (St Clair-Thomson and Sykes, 2010), and only a few studies indicate what is done with those falling at the median, usually adding them to the "low" group (e.g., Whaley, 2003; Hochwälder, 2009). If the field were to follow this convention, and assuming that the median of 80 can be justified, then this would equate to a consistency cut-off point of $>80$ on the EHI. As most handedness researchers use the EHI in a Likert scale format, this would equate to using the EHI85 system from the present study and it is suggested that this may be an appropriate way to create strength of preference categories. 
Examining direction of handedness influences, only a handful of studies have had a sufficiently large sample of consistent left-handers in order to carry out the "gold standard" analysis of comparing all four groups (Lyle et al., 2012a,b; current study). Unfortunately, this means that for the majority of the literature, the position of consistent left-handers is somewhat confused. In some cases they are dropped from analysis as there is "evidence that strong left- and strong right-handers differ from one another..." (Christman and Butler, 2011, p. 18), or that "strongly left-handed differ from both the strongly right- and the mixedhanded, and thus may constitute their own group" (Propper et al., 2005, p. 754). In other cases they are subsumed because "strong left-handers resemble strong right-handers, with mixed-handers being distinct from the other two strongly handed groups" (Christman et al., 2009, p. 1184). This ambiguity is clearly demonstrated in Prichard etal.'s (2013, p. 3) comprehensive review, where they argue that researchers should not consider direction, while paradoxically acknowledging that most of the studies in their review "compared ICH with CR-handers." Arguably, the clearest position to take is to conduct the "gold standard" test if at all possible, but should there not be enough left-handers to test for this, to clearly state that the difference is based on mainly CR-handers.

In terms of the handedness related differences it appears that both strength and direction of handedness may both relate to BIS/BAS. A relationship between left-handedness and behavioral inhibition has become quite well established, through behavioral studies (Wright et al., 2004, 2013; Wright and Hardie, 2011), selfreported measures (Wright et al., 2009; Lyle et al., 2012a; Hardie and Wright, 2013), comparative evidence (Cameron and Rogers, 1999; Rogers, 2009) and models of hemispheric specialization linking the left-hemisphere to avoidance and the right-hemisphere to approach (see Rutherford and Lindell, 2011 for a review). Previous research found that consistent handers showed significantly higher behavioral inhibition than $\mathrm{IH}$, which might be expected (e.g., Niebauer, 2004; Lyle et al., 2012a). When the present study examined the findings in terms of a relationship to direction of handedness as well (using EHI85) it clearly demonstrated that high scoring CL-handers have the highest mean BIS scores; that for left-handers regression analysis showed hand-strength was a significantly positive but weak predictor of BIS, and unsurprisingly BIS and hand strength were significantly correlated. Taken together, these findings suggest that for left-handers their relationship with behavioral inhibition links to degree of handedness in a way that is different from right-handers.

BAS-FS differences were also found, although these were in the opposite direction, with IH scoring higher than consistent. This is not surprising, as there is a body of evidence suggesting that IH are less conservative (Lyle and Grillo, 2014), more gullible (Christman et al., 2008), more open to non-standard ideas (Barnett and Corballis, 2002; Christman, 2013) and generally more risk aversive (Christman et al., 2007). Similar to the BIS findings, the main differences were largely driven by the influence of CL-handers being significantly different from all IH, but in this case, not CR-handers. This meant that regression analysis did not significantly differ between right- and left-handed groups. High BAS-FS has been linked to instant gratification and lack of future contemplation (Heym and Lawrence, 2010) and trait impulsivity (Smillie et al., 2006), and fits with behavioral evidence that lefthanders may show an initial response delay when confronted with novelty (Wright et al., 2013).

Putting this together, the current study shows that left-handers may be different in some aspects of personality, compared to right-handers. The extent to which this can be directly applied to other areas of personality is not currently clear, mainly due to the paucity of data on left-handers. Therefore, the present work will hopefully act as a catalyst for other researchers to collect data from a sufficient number of left-handers, so that future hand preference findings are driven by data, rather than assumptions. What can be generalized to other work is our overall finding that consistent leftand right-handers may not always behave in the same way. This is because it contrasts with other work which argues that direction is not important (Prichard et al., 2013), and potentially leaves a question as to how should the field proceed? The recent work of Lyle et al. (2012b) offers some insight here, as although they found consistent left- and right-handers did not differ in terms of memory accuracy, they did find that left-handers as a group were slower to make judgments about memory. In other work Propper et al. (2007) found that consistent left-handers had a different pattern of sleep compared to CR-handers, and Lyle et al. (2012a) found that CR-handers were more anxious than IH, but for lefthanders consistency did not relate to anxiety. As anxiety is seen as an outward sign of BIS activity (Corr and McNaughton, 2008) then the finding of Lyle et al. (2012a) resonates with the current results, and they give the intriguing possibility that direction may be important for left-handers and that strength may be important for right-handers.

The current research also has direct implications for the main theory for how hand strength may influence behavior. This theory relies upon the notion of an increased access to the righthemisphere for IH (compared to consistent handers), allowing them to better coordinate across both hemispheres, that is, having better interhemispheric interaction (Christman et al., 2004; Niebauer, 2004; Propper et al., 2005; Jasper et al., 2008). When taken from the viewpoint of right-handers these arguments are more or less the same, but by adding consistent left-handers to the equation then these become potentially separable issues.

Indeed, for left-handers the right-hemisphere access argument can also be questioned due to anatomical evidence. This suggests that there are structural asymmetries in the central sulcus, where the dorsolateral motor cortex of right-handers is larger in the left-hemisphere, while the opposite is found for left-handers (e.g., Amunts et al., 2000; Klöppel et al., 2010). Additionally, the contralateral motor control arrangement of the primary motor areas of the brain means that left-hand action is largely operationalized via the right-hemisphere (Grabowska et al., 2012), making increased right-hemisphere access arguments for mixed-handers untenable for left-handers. Also contrary to the "IH having an increased right-hemisphere access" model is work by Cherbuin and Brinkman (2006). Using Poffenberger's Paradigm, they found that for left-handers, increases in hand strength were related to increases in efficiency of interhemispheric interaction and as a group, strong left-handers had the highest accuracy (in letter-matching within and across visual 
fields), while strong right-handers had the lowest. On the other hand, arguments relating degree of handedness to interhemispheric interaction may be important. For example, Potter and Graves (1988) argued that CR-handers had a poorer interhemispheric transfer performance during a line drawing task when compared with non-right-handers. Luders etal. (2010) showed a negative association between corpus callosum size and strength of handedness, regardless of direction of handedness. This largely supports the idea that strength of handedness may demonstrate something important about how the hemispheres interact.

However, taking a wider examination of evidence, then it becomes apparent that compared to right-handers, left-handers appear to be more heterogeneous in terms of hemispheric organization and specialization (see Hervé etal., 2013, for a recent review). The assumption of consistent left-handers being similar to strong right-handers in interhemispheric connectivity is certainly open to debate, for example, Westerhausen etal. (2004) examined the corpus callosum, and found that left-handers had a higher density of fibers, suggesting greater interhemispheric connectivity. Other recent evidence examining motor control in the primary motor cortex, found that left-handers responded differently from right-handers when transcranial magnetic stimulation (TMS) was applied to either the dominant or non-dominant side (van den Berg et al., 2011). Right-handers were more disrupted in the task when the nondominant side was stimulated, but left-handers split into two distinct groups - one more disrupted by non-dominant side stimulation, the other by dominant side. A similar conclusion has recently been drawn by Lyle etal. (2012a, p. 13) who argue that "consistency-related effects on interhemispheric interaction may not be the same among left-handers as among right-handers." In their review, Hervé et al. (2013) make suggestions about future research questions, these include; investigating if left-handers as a group have a different neural organization than right-handers; do left-handers show variation in their intrinsic brain connectivity and how can structural and/or functional asymmetries be related to cognitive functioning in left-handers? Taken together, this suggests that the present theoretical underpinning of degree of handedness differences, while applicable to right-handers may need to be further investigated and/or re-evaluated when considering left-handers.

\section{LIMITATIONS}

The current study has some limitations, including the presentation of questionnaires using a web-based approach. By using this medium, there is the potential problem of participants not responding accurately or honestly. However, while this could occur within the dataset, there is no a priori reason to expect that the rate of error would differ according to handedness category, so the main findings should be robust. In addition, although webbased data was collected, the initial recruitment of participants was made before passing on the survey link, meaning that there was some degree of control of the process. It is therefore acknowledged that there will be an element of self-selection in terms of willingness to participate, but again there is no strong reason to believe that this would introduce a bias that would artificially create handedness based results. In order to improve accuracy from self-report questionnaires, future work should include either a pre-existing lie scale or add validity questions, to allow for removal of any clearly invalid responses (Fervaha and Remington, 2013). Finally, the regression results suggest that overall; strength of handedness is a very weak predictor of personality, while direction of handedness seems to demonstrate robust differences between left- and right-handers. This suggests the need for a much wider investigation of the validity of strength of handedness as a predictor.

\section{CONCLUSION}

The present study reinforces the view that consistent left- and right-handers do not always behave in the same way. The clear implication is that researchers need to gather sufficient data on consistent left-handers in order to delineate where behavior either converges with, or diverges from, right-handers. It also highlights the need for the handedness research community to be able to robustly defend the dichotomization of hand consistency on the basis of a strong theoretical and empirical evidence base, including an agreed split-point.

\section{REFERENCES}

Amunts, K., Jancke, L., Mohlberg, H., Steinmetz, H., and Zilles, K. (2000). Interhemispheric asymmetry of the human motor cortex related to handedness and gender. Neuropsychologia 38, 304-312. doi: 10.1016/S0028-3932(99) 00075-5

Barnett, K. J., and Corballis, M. C. (2002). Ambidexterity and magical ideation. Laterality 7, 75-84. doi: 10.1080/13576500143000131

Beratis, I. N., Rabavilas, A. D., Papadimitriou, G. N., and Papageorgiou, C. (2011). Eysenck's model of personality and psychopathological components in rightand left-handers. Pers. Individ. Differ. 50, 1267-1272. doi: 10.1016/j.paid.2010. 10.033

Bryden, M. P. (1977). Measuring handedness with questionnaires. Neuropsychologia 15, 617-624. doi: 10.1016/0028-3932(77)90067-7

Büsch, D., Hagemann, N., and Bender, N. (2010). The dimensionality of the Edinburgh handedness inventory: an analysis with models of the item response theory. Laterality 15, 610-628. doi: 10.1080/13576500903081806

Cameron, R., and Rogers, J. L. (1999). Hand preference of the common marmoset (Callithrix jacchus): problem solving and responses in a novel setting. J. Comp. Psychol. 113, 149-157. doi: 10.1037/0735-7036.113.2.149

Carver, C. S., and White, T. L. (1994). Behavioral inhibition, behavioral activation, and affective responses to impending reward and punishment: the BIS/BAS scales. J. Pers. Soc. Psychol. 67, 319-333. doi: 10.1037/0022-3514.67.2.319

Cherbuin, N., and Brinkman, C. (2006). Hemispheric interactions are different in left-handed individuals. Neuropsychology 20, 700-707. doi: 10.1037/08944105.20.6.700

Christman, S. D. (2013). Handedness and "earedness": strong right-handers are less likely to prefer obscure musical genres. Psychol. Music 41, 89-96. doi: $10.1177 / 0305735611415751$

Christman, S. D., and Butler, M. (2011). Mixed-handedness advantages in episodic memory obtained under conditions of intentional learning extend to incidental learning. Brain Cogn. 77, 17-22. doi: 10.1016/j.bandc.2011.07.003

Christman, S. D., Henning, B., Geers, A. L., Propper, R. E., and Niebauer, C. L. (2008). Mixed-handed persons are more easily persuaded and are more gullible: interhemispheric interaction and belief updating. Laterality 13, 403-426. doi: 10.1080/13576500802079646

Christman, S. D., Jasper, J. D., Sontam, V., and Cooil, B. (2007). Individual differences in risk perception versus risk taking: handedness and interhemispheric interaction. Brain Cogn. 63, 51-58. doi: 10.1016/j.bandc.2006.08.001

Christman,S. D., Propper, R. E., and Brown, T. J. (2006). Increased interhemispheric interaction is associated with earlier offset of childhood amnesia. Neuropsychology 20, 336-345. doi: 10.1037/0894-4105.20.3.336 
Christman, S. D., Propper, R. E., and Dion, A. (2004). Increased interhemispheric interaction is associated with decreased false memories in a verbal converging semantic associates paradigm. Brain Cogn. 56, 313-319. doi: 10.1016/j.bandc.2004.08.005

Christman, S. D., Sontam, V., and Jasper, J. D. (2009). Individual differences in ambiguous figure perception: degree of handedness and interhemispheric interaction. Perception 38, 1183-1198. doi: 10.1068/p6131

Cohen, J. (1983). The cost of dichotimization. Appl. Psychol. Meas. 7, 426-443. doi 10.1177/014662168300700301

Corr, P. J., and McNaughton, N. (2008). "Reinforcement sensitivity theory and personality," in The Reinforcement Sensitivity Theory of Personality, ed. P. J. Corr (Cambridge: Cambridge University Press), 155-187. doi: 10.1017/CBO9780511819384.006

Davidson, R. J. (1985). "Affect, cognition, and hemispheric specialization," in Emotions, Cognition, and Behavior, eds C. E. Izard and J. Kagan (New York: Cambridge University Press), 320-365.

Davidson, R. J. (1995). “Cerebral asymmetry, emotion and affective style," in Brain Asymmetry, eds R. J. Davidson and Hugdahl K. (Cambridge, MA: MIT Press), 361-387.

Davidson, R. J. (1998). Affective style and affective disorders: perspectives from affective neuroscience. Cogn. Emot. 12, 307-330. doi: 10.1080/026999398379628

DeCoster, J., Gallucci, M., and Iselin, A. R. (2011). Best practices for using median splits, artificial categorization, and their continuous alternatives. J. Exp. Psychopathol. 2, 197-209. doi: 10.5127/jep.008310

DeCoster, J., Iselin, A. R., and Gallucci, M. (2009). A conceptual and empirical examination of justifications for dichotomization. Psychol. Methods 14, 349-366 doi: 10.1037/a0016956

Dollfus, S., Alary, A., Razafimandimby, A., Prelipceanu, D., Rybakowski, J. K. Davidson, M., etal. (2012). Familial sinistrality and handedness in patients with first episode schizophrenia: the EUFEST study. Laterality 17, 217-224. doi: 10.1080/1357650X.2011.558510

Dragovic, M. (2004). Towards an improved measure of the Edinburgh Handedness Inventory: a one-factor congeneric measurement model using confirmatory factor analysis. Laterality 9, 411-419. doi: 10.1080/13576500342000248

Dragovic, M., Milenkovic, S., and Hammond, G. (2008). The distribution of hand preference is discrete: a taxometric examination. Br. J. Psychol. 99, 445-459. doi 10.1348/000712608X304450

Ellis, S. J., Ellis, P. J., and Marshall, E. (1988). Hand preference in a normal population. Cortex 24, 157-163. doi: 10.1016/S0010-9452(88)80025-X

Fazio, R., Coenen, C., and Denney, R. L. (2012). The original instructions for the Edinburgh Handedness Inventory are misunderstood by a majority of participants. Laterality 17, 70-77. doi: 10.1080/1357650X.2010.532801

Ferrucci, R., Brunoni, A. R., Parazzini, M., Vergari, M., Rossi, E., Fumagalli, M., et al. (2013). Modulating human procedural learning by cerebellar transcranial direct current stimulation. Cerebellum 12, 485-492. doi: 10.1007/s12311-0120436-9

Fervaha, G., and Remington, G. (2013). Invalid responding in questionnaire-based research: implications for the study of schizotypy. Psychol. Assess. 25, 1355-1360. doi: $10.1037 / \mathrm{a} 0033520$

Fox, N. A., Henderson, H. A., Rubin, K. H., Calkins, S. D., and Schmidt, L. A. (2001). Continuity and discontinuity of behavioural inhibition and exuberance: psychophysiological and behavioural influences across the first four years of life. Child Dev. 72, 1-21. doi: 10.1111/1467-8624.00262

Grabowska, A., Gut, M., Binder, M., Forsberg, L., Rymarczyk, K., and Urbanik A. (2012). Switching handedness: fMRI study of hand motor control in righthanders, left-handers and converted left-handers. Acta Neurobiol. Exp. 72, 439-451.

Gray, J. A., and McNaughton, N. (2000). The Neuropsychology of Anxiety: An Enquiry into the Functions of the Septo-hippocampal System, 2nd Edn. Oxford, UK: Oxford University Press.

Hardie, S. M., and Wright, L. (2013). The relationship between Revised Reinforcement Sensitivity Theory (rRST), handedness and indecision. Pers. Individ. Differ. 55, 312-316. doi: 10.1016/j.paid.2010.07.021

Hervé, P.-Y., Zago, L., Petit, L., Mazoyer, B., and Tzourio-Mazoyer, N. (2013). Revisiting human hemispheric specialization with neuroimaging. Trend Cogn. Sci. 17, 69-80. doi: 10.1016/j.tics.2012.12.004

Heym, N., and Lawrence, C. (2010). The role of Gray's revised RST in the Ppsychopathy continuum: the relationships of Psychoticism with a lack of fear and anxiety, and increased impulsivity. Pers. Individ. Differ. 49, 874-879. doi: 10.1016/j.paid.2010.07.021

Hochwälder, J. (2009). Burnout among Torgersen's eight personality types. Soc. Behav. Pers. 37, 467-480. doi: 10.2224/sbp.2009.37.4.467

Hopkins, W. D., and Bennett, A. J. (1994). Handedness and approach avoidance behavior in chimpanzees (Pan). J. Exp. Psychol. Anim. Behav. Process. 20, 413-418. doi: 10.1037/0097-7403.20.4.413

Irwin, J. R., and McClelland, G. H. (2003). Negative consequences of dichotomizing continuous predictor variables. J. Mark. Res. 40, 366-371. doi: 10.1509/jmkr.40.3.366.19237

Jackson, C. J. (2008). When avoidance leads to approach: how ear preference interacts with neuroticism to predict disinhibited approach. Laterality 13, 333-373. doi: 10.1080/13576500802063053

Jasper, J. D., Barry, K., and Christman, S. D. (2008). Individual differences in counterfactual production. Pers. Individ. Differ. 45, 488-492. doi: 10.1016/j.paid.2008.05.026

Jasper, J. D., Prothero, M., and Christman, S. D. (2009). I'm not sexist!!! Cognitive dissonance and the differing cries of mixed- and strong-handers. Pers. Individ. Differ. 47, 268-272. doi: 10.1016/j.paid.2009.03.010

Kaploun, K. A., and Abeare, C. A. (2010). Degree versus direction: a comparison of four handedness classification schemes through the investigation of lateralised semantic priming. Laterality 15, 481-500. doi: 10.1080/135765009 02958871

Kinsbourne, M. (1997). "Mechanisms and development of hemispecialization in children," in Handbook of Clinical Child Neuropsychology, 2nd Edn, eds E. FletcherJanzen and C. R. Reynolds (New York: Plenum), 102-119. doi: 10.1007/978-14757-5351-6_5

Kirveskari, E., Salmelin, R., and Hari, R. (2006). Neuromagnetic responses to vowels vs. tones reveal hemispheric lateralisation. Clin. Neurophysiol. 117, 643-648. doi: 10.1016/j.clinph.2005.11.001

Klöppel, S., Mangin, J.-F., Vongerichten, A., Frackowiak, R. S. J., and Siebner, H. R. (2010). Nurture versus nature: long-term impact of forced right-handedness on structure of pericentral cortex and basal ganglia. J. Neurosci. 30, 3271-3275. doi: 10.1523/JNEUROSCI.4394-09.2010

Knecht, S., Dräger, B., Deppe, M., Bobe, L., Lohmann, H., Flöel, A., et al. (2000). Handedness and hemispheric language dominance in healthy individuals. Brain 123, 2512-2518. doi: 10.1093/brain/123.12.2512

Luders, E., Cherbuin, N., Thompson, P. M., Gutman, B., Anstey, K. J., Sachdey, P., et al. (2010). When more is less: associations between corpus callosum size and handedness lateralization. Neuroimage 52, 43-49. doi: 10.1016/j.neuroimage.2010.04.016

Lyle, K. B., Chapman, L. J., and Hatton, J. L. (2012a). Is handedness related to anxiety? New answers to an old question. Laterality 18, 520-535. doi: 10.1080/1357650X.2013.783044

Lyle, K. B., Hanaver-Torrez, S. D., Hacklander, R. P., and Edlin, J. M. (2012b). Consistency of handedness, regardless of direction, predicts baseline memory accuracy and potential for memory enhancement. J. Exp. Psychol. Learn. Mem. Cogn. 38, 187-193. doi: 10.1037/a0024831

Lyle, K. B., and Grillo, M. C. (2014). Consistent-handed individuals are more authoritarian. Laterality 19, 146-163. doi:10.1080/1357650X.2013. 783044

Lyle, K. B., and Orsborn, A. E. (2011). Inconsistent handedness and saccade execution benefit face memory without affecting interhemispheric interaction. Memory 19, 613-624. doi: 10.1080/09658211.2011.595418

Lyle, K. B., McCabe, D. P., and Roediger, H. L. (2008). Handedness is related to memory via hemispheric interaction: evidence from paired associate recall and source memory tasks. Neuropsychology 22, 523-530. doi: 10.1037/0894-4105.22. 4.523

MacCallum, R. C., Zhang, S., Preacher, K. J., and Rucker, D. D. (2002). On the practice of dichotomization of quantitative variables. Psychol. Methods 7, 19-40. doi: 10.1037/1082-989X.7.1.19

McCullough, M. E., Cartera, E. C., DeWall, C. N., and Corrales, C. M. (2012). Religious cognition down-regulates sexually selected, characteristically male behaviors in men, but not in women. Evol. Hum. Behav. 33, 562-568. doi: 10.1016/j.evolhumbehav.2012.02.004

Milenkovic, S., and Dragovic, M. (2013). Modification of the Edinburgh Handedness Inventory: a replication study. Laterality 18, 340-348. doi: 10.1080/1357650X.2012.683196 
Moritz, S., Jacobsen, D., Willenborg, B., Jelinek, L., and Fricke, S. (2006). A check on the memory deficit hypothesis of obsessive-compulsive checking. Eur. Arch. Psychiatry Clin. Neurosci. 256, 82-86. doi: 10.1007/s00406-0050605-7

Niebauer, C. L. (2004). Handedness and the fringe of consciousness: strong handers ruminate while mixed handers self-reflect. Conscious. Cogn. 13, 730-745. doi 10.1016/0028-3932(92)90110-8

Oldfield, R. C. (1971). The assessment and analysis of handedness: the Edinburgh Handedness Inventory. Neuropsychologia 9, 97-113. doi: 10.1016/00283932(88)90084-X

Peters, M., and Murphy, K. (1992). Cluster analysis reveals at least three, and possibly five distinct handedness groups. Neuropsychologia 30, 373-380. doi 10.1016/0028-3932(92)90110-8

Potter, S., and Graves, R. (1988). Is interhemispheric transfer related to handedness and gender? Neuropsychologia 26, 319-325. doi: 10.1016/0028-3932(88)90084-X

Prichard E., Propper, R. E., and Christman, S. D. (2013). Degree of handedness, but not direction, is a systematic predictor of cognitive performance. Front. Psychol 4:9. doi: 10.3389/fpsyg.2013.00009

Propper, R. E., Christman, S. D., and Olejarz, S. (2007). Homerecorded sleep architecture as a function of handedness II: consistent rightversus consistent left-handers. J. Nerv. Mental Disord. 95, 689-692. doi: 10.1097/NMD.0b013e31811f44b8

Propper, R. E., Christman, S. D., and Phaneuf, K. A. (2005). A mixed handed advantage in episodic memory: a possible role of interhemispheric interaction. Mem. Cognit. 33, 751-757. doi: 10.3758/BF03195341

Rogers, L. J. (2009). Hand and paw preferences in relation to the lateralized brain. Phil. Trans. R. Soc. Biol. Sci. 364, 943-954. doi: 10.1098/rstb.2008.0225

Rose, J. P., and Nagel, B. (2012). Relation between comparative risk, absolute risk and worry: the role of handedness strength. J. Health Psychol. 18, 866-874. doi: $10.1177 / 1359105312456325$

Rutherford, H., and Lindell, A. (2011). Thriving and surviving: approach and avoidance motivation and lateralization. Emot. Rev. 3, 333-343. doi 10.1177/1754073911402392

Rydstedt, L. W., Cropley, M., Devereux, J. J., and Michalianou, G. (2008). The relationship between long-term job strain and morning and evening saliva cortisol secretion among white-collar workers. J. Occup. Health Psychol. 13, 105-113. doi: 10.1037/1076-8998.13.2.105

Schmidt, L. A., Fox, N. A., Schulkin, J., and Gold, P. W. (1999). Behavioral and psychophysiological correlates of self-presentation in temperamentally shy children. Dev. Psychobiol. 35, 119-135. doi: 10.1002/(SICI)10982302(199909)35:2<119::AID-DEV5>3.0.CO;2-G

Shackman, A. J., McMenamin, B. W., Maxwell, J. S., Greischar, L. L., and Davidson, R. J. (2009). Right dorsolateral prefrontal cortical activity and behavioral inhibition. Psychol. Sci. 20, 1500-1506. doi: 10.1111/j.1467-9280.2009.02476.x

Smillie, L. D., Jackson, C. J., and Dalgleish, L. I. (2006). Conceptual distinctions among Carver and White's (1994) BAS scales: a reward-reactivity versus trait impulsivity perspective. Pers. Individ. Differ. 40, 1039. doi: 10.1016/j.paid.2005.10.012

Smith, D. S., Jones, B. C., and Allan, K. (2013).Socio-sexuality and episodic memory function in women: further evidence of an adaptive "mating mode". Mem. Cognit. 41, 850-861. doi: 10.3758/s13421-013-0301-1

St Clair-Thomson, H., and Sykes, S. (2010). Scoring methods and the predictive ability of working memory tasks. Behav. Res. Methods 42, 969-975. doi: 10.3758/BRM.42.4.969

Streiner, D. L. (2002). Breaking up is hard to do: the heartbreak of dichotomizing continuous data. Can. J. Psychiatry 47, 262-266.

Sutton, S. K., and Davidson, R. J. (1997). Prefrontal brain asymmetry: a biological substrate of the behavioral approach and inhibition systems. Psychol. Sci. 8, 204 210. doi: 10.1111/j.1467-9280.1997.tb00413.x
Tops, M., and Bokshem, M. A. S. (2011). Cortisol involvement in mechanisms of behavioral inhibition. Psychophysiology 48, 723-732. doi: 10.1111/j.14698986.2010.01131.x

van den Berg, F. E., Swinnen, S. P., and Wenderoth, N. (2011). Involvement of the primary motor cortex in controlling movements executed with the ipsilateral hand differs between left and right-handers. J. Cogn. Neurosci. 23, 3456-3469. doi: 10.1162/jocn_a_00018

Veale, J. (2014). Edinburgh Handedness Inventory - Short Form: a revised version based on confirmatory factor analysis. Laterality 19, 164-177. doi: 10.1080/1357650X.2013.783045

Westerhausen, R., Kreuder, F., Dos Santos, S. S., Walter, C., Woerner, W., Wittling, R. A., et al. (2004). Effects of handedness and gender on macro- and microstructure of the corpus callosum and its sub regions: a combined high resolution and diffusion-tensor MRI study. Cogn. Brain Res. 21, 418-426. doi: 10.1016/j.cogbrainres.2004.07.002

Westfall, J., Jasper, J. D., and Christman, S. D. (2012). Inaction inertia, the sunk cost effect, and handedness: avoiding the losses of past decisions. Brain Cogn. 80, 192-200. doi: 10.1016/j.bandc.2012.06.003

Westfall, J., Jasper, J. D., and Zelmanova, Y. (2010). Differences in time perception as a function of strength of handedness. Pers. Individ. Differ. 49, 629-633. doi: 10.1016/j.paid.2010.05.036

Whaley, A. L. (2003). Confluent paranoia in African American psychiatric patients: an empirical study of Ridley's typology. J. Abnorm. Psychol. 111, 568-577. doi: 10.1037/0021-843X.111.4.568

Williams, S. M. (1986). Factor analysis of the Edinburgh Handedness Inventory. Cortex 22, 325-326. doi: 10.1016/S0010-9452(86)80058-2

Wright, L., and Hardie, S. M. (2011). "Not ready to sort it yet". Revised Reinforcement Sensitivity Theory (rRST) predicts left-handed behavioural inhibition during a manual sorting task. Laterality 16, 753-767. doi: $10.1080 / 1357650$ X.2010.521752

Wright, L., and Hardie, S. M. (2012). Are left handers really more anxious? Laterality 17, 629-642. doi: 10.1080/1357650X.2011.615126

Wright, L., Hardie, S. M., and Rodway, P. (2004). Pause before you respond: handedness influences response style on the Tower of Hanoi task. Laterality 9, 133-147. doi: 10.1080/13576500244000265

Wright, L., Hardie, S. M., and Wilson, K. (2009). Handedness and behavioural inhibition: left-handed females show most inhibition as measured by BIS/BAS self-report. Pers. Individ. Differ. 46, 20-24. doi: 10.1016/j.paid.2008. 08.019

Wright, L., Watt, S., and Hardie, S. M. (2013). Influences of lateral preference and personality on behavior toward a manual sorting task. Pers. Individ. Differ. 54, 903-907. doi: 10.1016/j.paid.2013.01.005

Conflict of Interest Statement: The authors declare that the research was conducted in the absence of any commercial or financial relationships that could be construed as a potential conflict of interest.

Received: 06 September 2013; accepted: 01 February 2014; published online: 20 February 2014.

Citation: Hardie SM and Wright L (2014) Differences between left-and right-handers in approach/avoidance motivation: influence of consistency of handedness measures. Front. Psychol. 5:134. doi: 10.3389/fpsyg.2014.00134

This article was submitted to Cognition, a section of the journal Frontiers in Psychology. Copyright $\odot 2014$ Hardie and Wright. This is an open-access article distributed under the terms of the Creative Commons Attribution License (CC BY). The use, distribution or reproduction in other forums is permitted, provided the original author(s) or licensor are credited and that the original publication in this journal is cited, in accordance with accepted academic practice. No use, distribution or reproduction is permitted which does not comply with these terms. 